\title{
Tratamento da doença de Kienbock com colapso cárpico (Lichtman IIIb) com osteotomia de encurtamento do radio - 16 casos
}

\author{
J. C. Botelheiro, S. Silverio \\ Unidade de Cirugia da Mão, Hospital de Santana. Parede. Portugal.
}

\begin{abstract}
Resumo: Objectivos. Rever os resultados das osteotomias de encurtamento do rádio efectuadas nos doentes com doença de Kienbock avançada (estadio IIIB).

Material e métodos. Nos últimos 24 anos operámos 50 casos de doença de Kienbock com osteotomia de encurtamento do rádio. 16 tinham já colapso cárpico (Lichtman IIIB). Os seus processos clínicos e radiografias foram revistos.

Resultados. Os bons resultados clínico obtidos em doentes só com colapso do semi-lunar (Lichtman IIIA) confirmam os da literatura, mas obtivemos resultados igualmente bons em casos já com colapso cárpico (Lichtman IIIB), que apresentamos. Conclusões. Os resultados aqui apresentados poderiam alargar as clássicas indicações da osteotomia de encurtamento do rádio no tratamento da doença de Kienbock
\end{abstract}

Palabras claves: Kienbock, colapso cárpico, osteotomia rádio.

\section{Treatment of advanced Kienbock disease (Lichtman IIIB) with a shortening osteotomy of the radius - 16 cases}

\begin{abstract}
Objectives. To review the results of shortening osteotomies of the radius in IIIB Kienbock disease patients. Material and Method. In the last 24 years we treated 50 cases of Kienbock disease by a shortening osteotomy of the radius. 16 of them had already carpal collapse. All patients charts and x-rays were reviewed.

Results. Good post-operative results in patients only with lunate collapse (Lichtman IIIA) confirm those presented by many authors, but we present equally good results in cases already with carpal collapse (Lichtman IIIB).

Conclusions. The results presented here could enlarge the current indications for shortening osteotomies of the radius in the treatment of Kienbock's disease.
\end{abstract}

Key words: Kienbock disease, carpal colapse, radial osteotomy.

A doença de Kienbock - necrose avascular do semi -lunar - descrita em 1910, foi classificada em estadios radiológicos progressivos por Stahl e Decoulx! Nos estadios de Decoulx I e II o semi-lunar mantém a sua forma original, embora com alterações da sua densidade radiológica, no estadio III aparece já um achatamento do semi-lunar e no estadio IV existem já sinais radiológicos de artrose rádio-cárpica - osteofitose e estreitamento da interlinha articular. Lichtman mais tarde dividiu o estadio III em "A" - sem colapso cárpico - e "B" - já com colapso cárpico, com implicações terapêuticas².

Aparte o tratamento conservador - imobilização - com resultados insuficientes ${ }^{3-5}$ - muitas técnicas cirúrgicas foram usadas no tratamento da doença de Kienbock 6 .

De notar que recentemente foram introduzidas classificações da doença de Kienbock com base nos aspectos da Ressonância Magnética ${ }^{6}$ e da artroscopia ${ }^{7}$, esta última com implicações terapêuticas teóricas.
As osteotomias de alongamento do cúbito ou de encurtamento do rádio foram iniciadas pelo trabalho de Hulten que em 1928' mostrou que o "cubitus minus" era mais frequente nos doentes de Kienbock que na população em geral e portanto seria uma das causas da doença.

Embora isso tenha sido posto em causa ${ }^{8}$, estudos biomecânicos parecem provar que essas cirurgias de facto descomprimem o semi-lunar ${ }^{9}$ e a osteotomia de encurtamento do rádio, sem os problemas de consolidação do alongamento do cúbito ${ }^{10}$, tornou-se provavelmente a forma de tratamento mais popular da doença de Kienbock ${ }^{1,6,11-16}$, por vezes combinada com outras técnicas ${ }^{17,18}$.
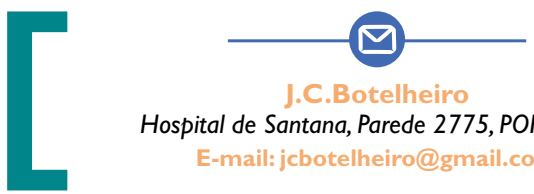

Hospital de Santana, Parede 2775, PORTUGAL E-mail: jcbotelheiro@gmail.com 
Artrodeses intercárpicas como a STT foram aconseIhadas na doença de Kienbock com colapso cárpico (estadio IIIB) e também no estadio IIIA sem "cubitus minus", porque se consideraram sem indicação teórica para uma osteotomia de encurtamento do rádio 2,19,20. Para estes últimos doentes outras técnicas cirúrgicas foram também propostas - osteotomias angulares ${ }^{6,21}$ ou simplesmente oblíquas do rádio ${ }^{22,23}$ e uma osteotomia de encurtamento do grande Osso $^{24}$; e a revascularização do semi-lunar ${ }^{25,26}$.

Por outro lado, as próteses do semi-lunar deram maus resultados a longo termo ${ }^{27}$ e a carpectomia proximal ou a artrodese rádio-cárpica são geralmente consideradas cirurgias de resgate ${ }^{2}$ como a desnervação do punho ${ }^{28}$.

\section{MATERIAL E MÉTODO}

Desde 1985, 50 osteotomias de encurtamento do rádio foram realizadas em doenças de Kienbock na Unidade de Cirurgia da Mão do nosso Hospital.

Todos os processos dos doentes foram revistos, mas apenas os 16 doentes com colapso cárpico (Lichtman IIIB) são aqui apresentados. Embora a todos os doentes fosse pedido para voltarem anualmente à nossa consulta, muitos, vivendo longe, não o fizeram - a última revisão clínica e radiológica foi entre os 9 meses e 17 anos após a cirurgia (média 4 anos e 7 meses).

Apenas 5 eram do sexo masculino. A idade oscilava entre os 15 e os 66 anos, com uma média de 31,4 anos. Apenas 5 punhos esquerdos foram operados, nenhum bilateral.

As suas queixas duravam desde I mês a 3 anos.

A dôr pré-operatória foi classificada como:

Grau 3 - constante - 3 casos

Grau 2 - mesmo em trabalhos leves - II

Grau I - só em trabalho pesado - I

Grau 0 - indolor - nenhum.

Pre-operatoriamente, a mobilidade do punho em flexão-extensão era em média de $68^{\circ}$ e a força de preensão, medida com um manómetro de pera e comparada com a mão contrária, tinha uma média de $21 \%$.
Estas medidas não foram efectuadas num doente espástico ${ }^{29}$.

A variância cubital, em $R X$ póstero-anterior do punho, foi classificada como: "cubitus minus" - I I doentes (1) Figura | variância cubital neutra- 58 doentes tinham fragmentação do semi-lunar visível nas radiografias.

As osteotomias de encurtamento do rádio (2 a 3mm) foram feitas nos primeiros anos por via dorsal de Thompson na metáfise distal em 2 casos, fixadas com uma placa recta (4) Figura 2. Os últimos 14 casos foram operados por via palmar com uma placa em T e imobilizados com uma tala antebráquio-palmar durante 4 semanas. Os doentes mobilizavam então o punho activamente durante I mês e eram depois enviados para fisioterapia durante 4 a 6 semanas antes de retomarem o seu trabalho quando a osteotomia estava consolidada.

Num doente muito queixoso e com um claro sindroma de túnel cárpico foi efectuada uma neurotomia do interósseo posterior e uma retinaculotomia palmar ao mesmo tempo que a osteotomia.

\section{RESULTADOS}

Todos estes doentes, de acordo com a nossa revisão retrospectiva dos seus processos, estavam melhor após a cirurgia que antes.

A dor, geralmente classificada nos graus 2 ou 3 antes da cirurgia (media 2, I4), era normalmente I ou 0 após (média 0,67); a flexão-extensão média do punho passou de $68^{\circ}$ para $94^{\circ}$ e a força de preensão de $21 \%$ para $73 \%$ do punho contralateral @ Figura 3.

Usando o exigente "Mayo Wrist Score" a pontuação média pré-operatória dos casos operados era de 24 pontos (todos "maus") passando a 68 pontos de média no post-operatório - I resultado "excelente", 4 "bons" e 10 "satisfatórios". Na classificação clínica de Nakamura27 obtivemos II resultados "excelentes", 4 bons e I "medíocre".

Portanto, a osteotomia de encurtamento do radio, classicamente aconselhada apenas nos estadios I a IIIA $\mathrm{A}^{2}$, deu-nos bons resultados clínicos em doentes já com colapso cárpico (estadio IIIB), aliás muito semeIhantes aos obtidos por nós no estadio IIIA". 

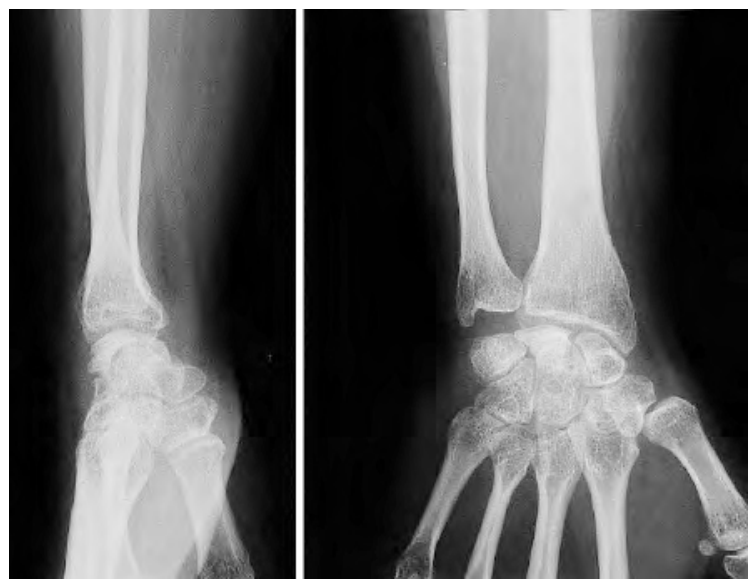

Figura I. Radiografia pré-operatória de doença de Kienbock estadio IIIB e variância cubital neutra.

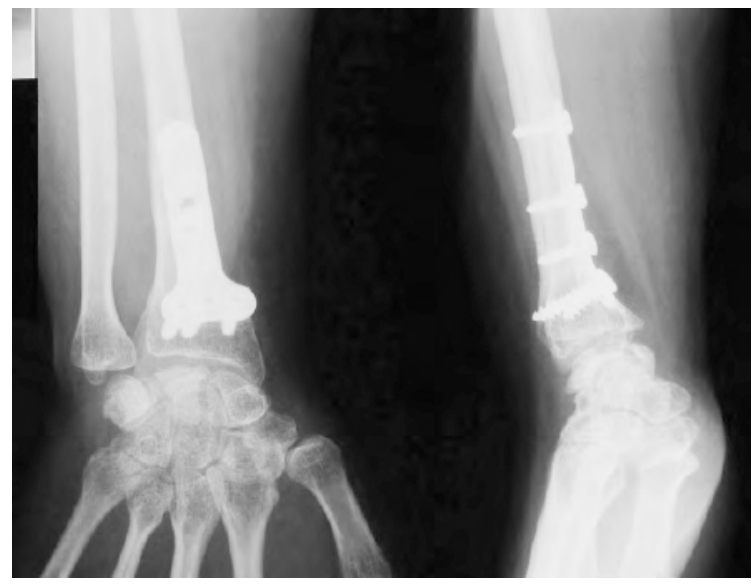

Figura 2. Radiografia do mesmo caso 10 anos após a cirurgia.

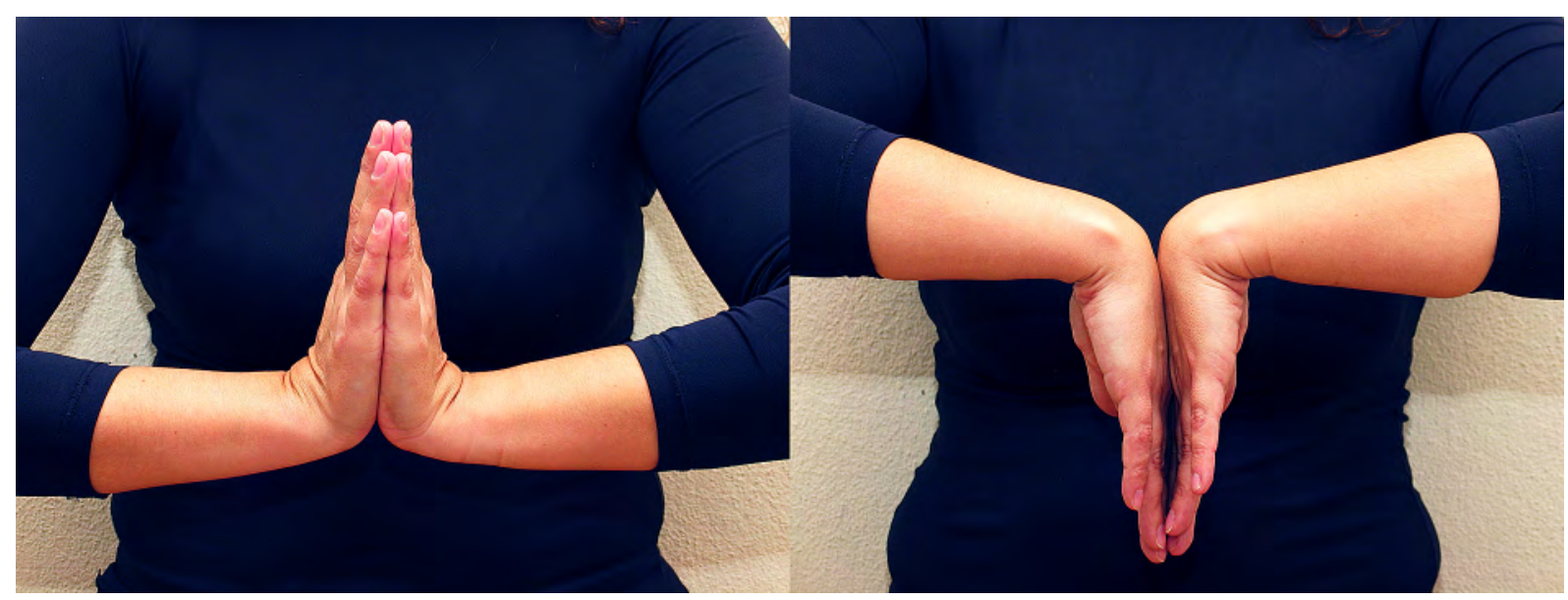

Figura 3. Mobilidade actual do mesmo caso.

Os resultados radiologicos não foram tão bons de uma forma bastante subjectiva, considerando o aspecto do semi-lunar e sinais de artrose, as radiografias post-operatórias foram consideradas melhoradas apenas em 2 doentes, não melhoradas em 6 e piores em 7 casos.

Apenas os 2 doentes com placas dorsais foram reoperados para a sua extracção e num deles foi nessa altura efectuada uma neurotomia do interósseo posterior.

\section{DISCUSSÃO}

A doença de Kienbock é de etiologia desconhecida e a sua evolução sem tratamento poderá não ser sempre má, pois a artrose do punho sintomática causada pela necrose avascular do punho é raramente vista na prática clínica ${ }^{3}$. Mais uma razão para se evitarem no seu tratamento cirurgias agressivas, como as artrodese intercárpicas.

O encurtamento dum rádio que não é mais longo que o cúbito parece ilógico porque não corrigiria uma causa da doença e poderia levar a um conflito cúbitocárpico ou dores rádio-cubitais, embora isso só tenha sido descrito em encurtamentos do rádio superiores aos aqui descritos ${ }^{12}$. Não tivemos casos de dôr cubital duradoura na nossa série, mesmo nos doentes com variância cubital neutra pré-operatória. E os nossos resultados são semelhantes nos casos com "cubitus minus" e nos outros - provavelmente o importante não é "curar" a causa da doença mas apenas descomprimir o semi-lunar.

Nos doentes no estadio IIII com um escafoide horizontalizado em relação ao punho contalateral, muitos 
autores aconselham uma artrodese intercárpica que recolocaria o escafoide na sua posição correcta e descomprimiria o semi-lunar ${ }^{2,30}$, mas estas cirurgias limitam seriamente a mobilidade do punho 19,20,30.

\section{CONCLUSIÓN}

Como outros resultados publicados, os nossos parecem indicar que uma osteotomia de encurtamento do rádio, fixada com uma placa palmar, é na doença de Kienbock uma técnica com poucas complicações e resultados bons e duradouros ${ }^{31}$, não só nos estadios II e IIIA, mas também no estadio IIIB ${ }^{32,33}$, talvez porque o colapso cárpico na doença de Kienbock não leva tão rapidamente a uma artrose rádio-cárpica como nos casos pós-traumáticos ${ }^{34}$. A causa poderá ser que o escafóide e o semi-lunar permanecem solidários na doença de Kienbock ${ }^{35}$, sem movimentos anormais.

A osteotomia de encurtamento do rádio parece especialmente indicada em doentes com trabalho leve e que necessitem de uma boa mobilidade do punho.

Finalmente, não impede a realização de qualquer cirurgia mais radical - artrodese intercárpica ou do punho, por exemplo - posteriormente, em caso de insucesso.

\section{CONFLITOS DE INTERESSE}

Os autores declaram não haver conflitos de interesse.

\section{BIBLIOGRAFÍA}

I. Axelson R, Moberg E. Le traitement de la maladie de Kienbock et le role des interventions de reequilibration radio-cubitale. En: Razemon JP, Fisk GR (Eds.) Le poignet. Paris: Expansion Scientifique Francaise; 1983. p. 21 0-7.

2. Alexander AH, Lichtman DM.The Kienbock dilemma - how to cope. En: Nakamura R, Linscheid RL, Miura $T$ (Eds.) Wrist Disorders - current concepts and challenges. Tokyo: Springer-Verlag; 1992. p. 7986.

3. Rasmussen F, Schantz K. Radiological aspects of lunatomalacia. Eur J Radio. 1987;7:199-202.

4. Salmon J, Stanley JK, Trail IA. Kienbock's disease Conservative treatment versus radial shortening. I Bone Joint Surg Br. 2000;82:820-3.
5. Mikkelsen SS, Gelinek J. Poor function after nonoperative treatment of Kienbock's disease. Acta Orthop Scand. 1987;58:24I-3.

6. Lamas C. La enfermedad de Kienbock. Barcelona: J.M.Bosch Editor; 2005.

7. Bain Gl, Begg M. Arthroscopic assessment and classification of Kienbock's disease. Tech Hand Up Extrem Surg. 2006; I O( I): 8- I3.

8. d'Hoore K, DeSmet L, Verelen K et al. Negative ulnar variance is not a risk factor for Kienbock disease. J Hand Surg Am. 1994; 19:229-31.

9. Trumble T, Glisson R, Seaber AV, Urbaniak JR. A biomechanical comparison of the methods for treating Kienbock's disease. J Hand Surg Am. | 986; | | :88-93.

10. Trail IA, Linscheid RL, Quenzer DE, Sherer PA. UInar lengthening and radial recession procedures for Kienbock's disease - long-term clinical ang radiographic follow-up. J Hand Surg Br. 1996;2 I: I6976.

I I. Botelheiro JC. La osteotomia de acortamiento del rádio en el tratamiento de la enfermedad de Kienbock - analisis de una serie de 20 casos. Rev Esp Cir Mano. 1996;51:7-15.

12. Nakamura R, Imaeda T et Miura T: Radial shortening for Kienbock's disease: factors affecting the operative result. J Hand Surg Am. 1990; 1 5B:40-4.

13. Razemon JP. La maladie de Kienbock - etude radiologique et therapeutique a propos de 22 cas de racourcissement du radius. En: Razemon JP, Fisk GR (Eds.) Le poignet, Paris: Expansion Scientifique Francaise; 1983. p. 204-9.

14. Gomis R, Martin B, Idoux, Chamas M, Allieu Y. Maladie de Kienbock - traitement par osteotomie de racourcissement du radius. Rev Chir Orthop. 1994:80: 196-204.

15. Siala A, Aieche MLB, Frikha R, Ghannouchi G, Moula $T$. Resultats du racourcissement diaphysaire du radius dans le traitementde la maladie de Kienbock. Rev Chir Orthop. 2000;86:1 5 I-7.

16. Weiss A-PC, Weiland AJ, Moore JR et al. Radial shortening for Kienbock's disease.J Bone Joint Surg Am. 1991;73: 384-91.

17. Quenzer DE, Dobyns JH, Linscheid RL, Trail IA, Vidal MA. Radial recession osteotomy for Kienbock's disease. J Hand Surg Am. 1997;22:386-95.

18. Mathoulin C, Wahegaonkar AL. Revascularisation of the lunate by a volar vascularised bone graft and an osteotomy of the radius in the treatment of the Kienbock's disease. Microsurgery. 2009;29:373-8. 
19. Condit DP, Idler RS, FischerTJ, Hastings II H. Preoperative factors and outcome after lunate decompression for Kienbock's disease. J Hand Surg Am. 1994; | 8:69|-6

20. Voche P, Bour C, Merle M. Scapho-trapeziotrapezoid arthrodesis in the treatment of Kienbock's disease: a study of 16 cases. J Hand Surg Br. | 992; 17:5-II.

21. Watanabe K, Nakamura R, Horii E et al. Biomechanical analysis of radial wedge osteotomy for the treatment of Kienbock's disease. J Hand Surg Am. 1993; | 8:686-90.

22. Wijffels MME, Jupiter J. Radial oblique osteotomy in the treatment of Kienbock disease. Curr Orthop Pract. 2009;2:593-7.

23. Blanco RH, Blanco FR. Osteotomy of the radius without shortening for Kienbock disease: a 10 year follow-up. J Hand Surg Am. 20 12;37A: 222 I-5.

24. Almquist EE. Capitate shortening osteotomy. En: Blair WF, Steyers CM (Eds.) Techniques in hand surgery. Philadelphia:Williams and Wilkins; 1996. p. 1067.

25. Bishop AT. Vascularised pedicle grafts from the dorsal distal radius: design and application for carpal pathology. En: Saffar P, Amadio PC, Foucher G. (Eds.) Current practice in hand surgery. London: Martin Dunitz Ltd; 1997. p. 307-I3.

26. Bochud RC, Buchler U. Kienbock's disease, early stage 3 - height reconstruction and core revascularization of the lunate. I Hand Surg $\mathrm{Br}$. 1994; 19:466-77.
27. O'Flanagan SJ, Curtin J. Lunate silastic arthroplasty in Kienbock's disease. J R Coll Surg Edin. 1992;37:52-6.

28. Foucher G, da Silva JB, Ferreres A. La denervation totale du poignet. A propos de 50 cas. Rev Chir Orthop. 1992;78: 186-90.

29. Rooker GD, Goodfellow JW. Maladie de Kienbock chez les infirmes moteurs cérébraux. En: Razemon JP, Fisk GR (Eds). "Le poignet". Paris: Expansion cientifique Française; 1983.

30. Inoue G. Capitate-hamate fusion for Kienbock's disease: Good results in 8 cases followed for 3 years. Acta Orthop Scand. 1992;63:560-2.

3I. Watanabe T, Takahara M, Tsuchida $\mathrm{H}$ et al. Longterm follow-up of radial shortening osteotomy for Kienbock disease. J Bone Joint Surg Am. 2008;90: | 705-। I.

32. Iwasaki N, Minami A, Oizumi N, Suenaga N, Kato $H$, Minami M. Radial osteotomy for late stage Kienbock's disease. J Bone Joint Surg Br. 2002;84:673-7.

33. Calfee RP, Van Steyn MO, Gyuricza $C$ et al. Joint leveling for advanced Kienbock's disease. J Hand Surg Am. 201 0;35A: 1947-54.

34. Tanigushi Y, Tamaki T, Honda T, Yoshida M. Rotatory subluxation of the scaphoid in Kienbock's disease is not a cause of scaphlunate advanced collapse (SLAC) in the wrist. J Bone Joint Surg Br. 2002;84:684-91.

35. Dias JJ, Lunn P.Ten questions on Kienbock's disease of the lunate. J Hand Surg Am. 20 I0;35:538-43. 